\title{
Atomic-Scale Investigations of Charge-lattice Modulation in a Hole-doped Charge- ordered Ferrite
}

Shiqing Deng ${ }^{1}$, Lijun $\mathrm{Wu}^{2}$, Hao Cheng ${ }^{3}$, Jin-Cheng Zheng ${ }^{3}$, Shaobo Cheng ${ }^{2}$, Jun $\mathrm{Li}^{2}$, Wenbi Wang ${ }^{4}$, Jian Shen ${ }^{4}$, Jing $\mathrm{Tao}^{2}$, Jing $\mathrm{Zhu}^{5}$ and Yimei $\mathrm{Zhu}^{2}$

${ }^{1}$ University of Science and Technology Beijing, Beijing, Beijing, China (People's Republic), ${ }^{2}$ Brookhaven National Laboratory, Upton, New York, United States, ${ }^{3}$ Xiamen University, Xiamen, Fujian, China (People's Republic), ${ }^{4}$ Fudan University, Shanghai, Shanghai, China (People's Republic), ${ }^{5}$ Tsinghua University, Beijing, Beijing, China (People's Republic)

In charge-ordered phases, broken symmetry in quantum states is widely considered to breed fascinating physics and broad potential applications. Often, such symmetry breaking in quantum states would result in intimately coupled electronic and lattice periodic modulation [1]. This charge-lattice modulation can be very complicated due to the close couplings between various degrees of freedom. Especially when ubiquitous singularities and solutions exist, the picture is further complicated and, in the meantime, emergent modulations can be generated [2]. Questions then arise as to how the charge-lattice modulation structure would be altered by the existence of these singularities and solitons. Lacking accurate descriptions of such modulations would lead to an incomplete understanding, preventing us from delving into the underlying physics. Hence accurately probing modulations in the charge-ordered systems, especially the ones with singularities, in both electronic state and atomic lattice, is of great importance to the community, allowing disentanglement of the intertwined various degrees of freedom.

Here, by purposely introducing excess holes in a prototype charge-ordered system $\mathrm{LuFe}_{2} \mathrm{O}_{4}+\delta$, we present a new type of charge-lattice incommensurate modulation (ICM) structure with quasiperiodic singularities in both periodic lattice displacements (PLDs) and charge distribution. This new modulation structure manifests marked differences from the traditional one and requires significant modifications to the conventional modeling of ordered structures. Using state-of-the-art electron microscopy, we directly map picometer scale periodic lattice displacements in $\mathrm{LuFe}_{2} \mathrm{O}_{4+\delta}$ (Fig. 1(e) and (f)). Stripes with positive and negative displacement alternatively distribute along [027] ${ }^{*}$ direction yielding a periodicity of around 10 $\AA(\lambda=1 /|\mathbf{q} \mathbf{p}|)$, which corresponds to the primary modulation $(\mathrm{PM})$ wave vector $\mathbf{q} \mathbf{p}(\sim 0.135 \mathbf{g} \mathbf{1})$. More importantly, displacement stripes are found periodically glide along (01-7) planes, caused by singularities in the PM wave phase. Periodic singularities in phase and oscillation in amplitude generate a second-order modulation $(\mathrm{SOM})$ with the wave vector of $\mathbf{q}_{\mathbf{s}}\left(\sim 0.110 \mathbf{g}_{2}\right)$. Furthermore, atomically resolved electron energy-loss spectroscopy (EELS) investigations (Fig. 1(d)) reveal the analogous singularities and oscillation in the charge distributions caused by doped holes (Fig. 1(g)), which clarify the origin for the abnormal lattice modulation. Therefore, this new charge-lattice modulation essentially manifests the feature that the phase and amplitude of the primary wave $\mathbf{P}(\mathbf{q p}, \mathbf{r})$ are modulated by a phase function and an amplitude function, respectively, forming a new secondary modulation (Fig. 2(a)). Based on these quantitative microscopy investigations, a new type of modulation wave that addresses singularities is defined, where additional degrees of freedom are added in both phase and amplitude parameter spaces,

$$
\mathbf{M}\left(\mathbf{q}_{\mathbf{p}}, \mathbf{q} \mathbf{s}, \mathbf{r}\right)=\mathbf{A}\left(\mathbf{q}_{\mathbf{s}}, \mathbf{r}\right) \sin \left[2 \pi \mathbf{q}_{\mathbf{p}} \cdot \mathbf{r}+\phi\left(\mathbf{q}_{\mathbf{s}}, \mathbf{r}\right)\right],
$$

$\mathbf{q}$ is the wave vector and $\mathbf{r}$ is the atom position. Moreover, direct electron diffraction calculations of ICM based on Bloch wave method have been conducted to underline the essential differences between this 
SOM modulation structure and traditional two-dimensional modulation structure (Fig. 2(b) and 2(c)) [3]. The newly developed modulation formulism in this study provides a more accurate and universal depiction of the order parameter and can be widely applicable to numerous ordered systems, as singularities are ubiquitous. The insights from this work advance our understanding of the nature of singularity and modulation, as well as veiled entanglements between various degrees of freedom [4,5].
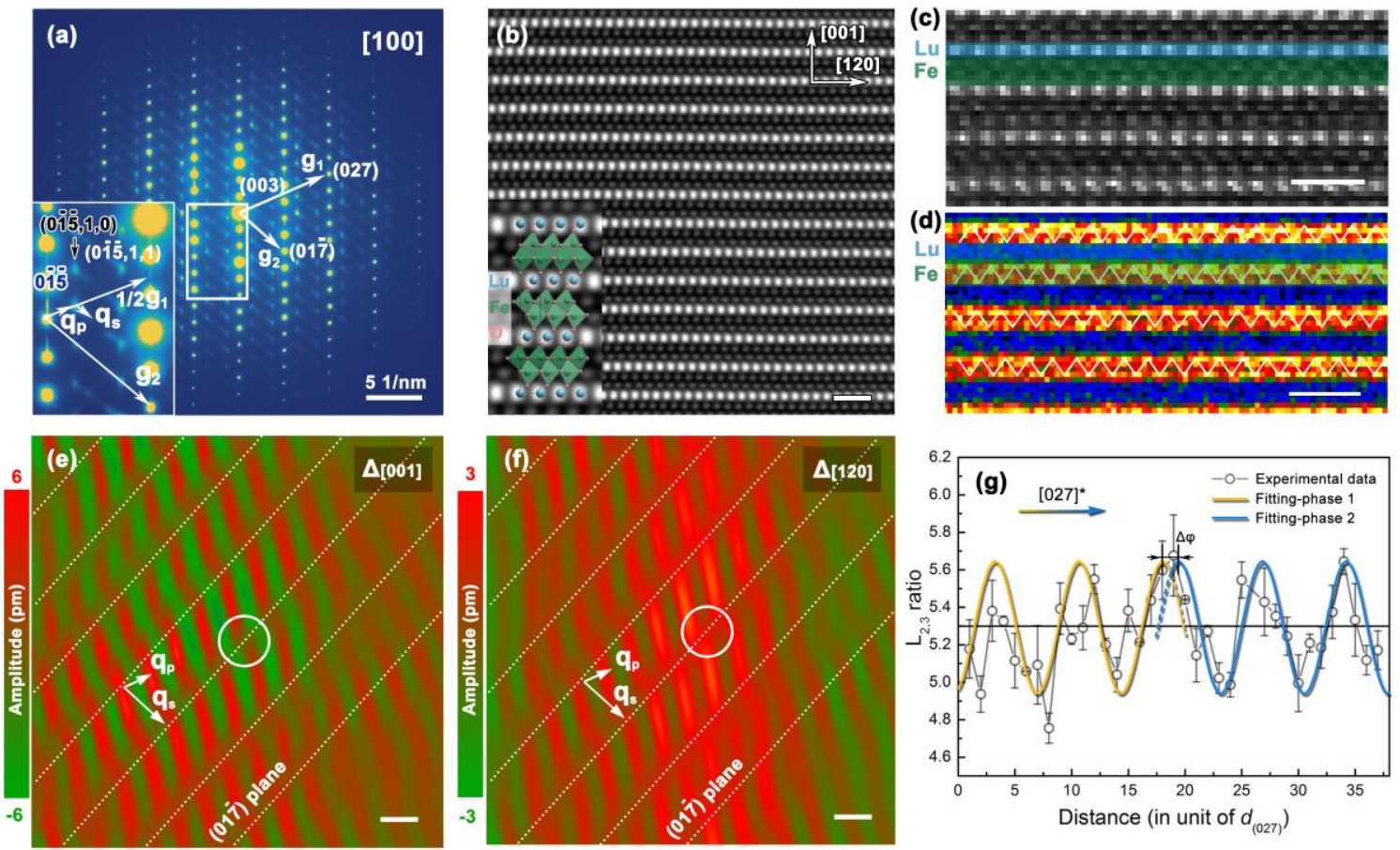

Figure 1. Second-order incommensurate modulation in $\mathrm{LuFe} 2 \mathrm{O} 4+\delta$. (a) [100] zone axis electron diffraction pattern showing a series of satellite reflections. (b) HAADF-STEM image along the a-axis showing distorted atomic planes. (c) HAADF-STEM image simultaneously acquired with the spectrum image. (d) Atomically resolved spectroscopic data of Fe-L2,3 edges. (e) and (f) PLD map along the [001] (e) and [120] direction (f), showing periodic phase singularities. (g) Integration of L3/L2 ratios at each (027) plane. The scale bars are $1 \mathrm{~nm}$.
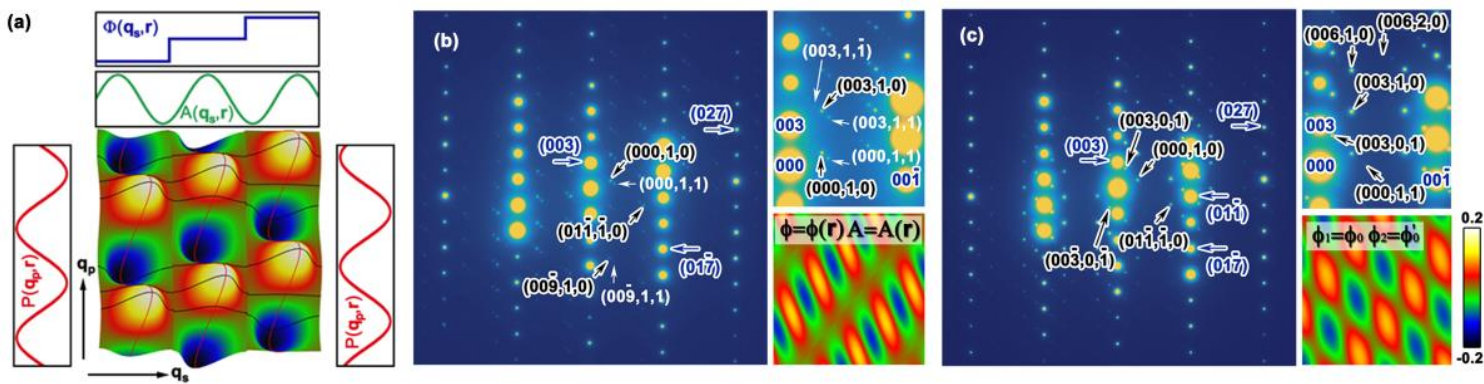

Figure 2. Comparison between the second-order modulation and traditional 2-D modulation. (a) A schematic model illustrating the essential characteristics of second-order modulation. (b) Second-order modulation calculated based on Eqn. (1), which is consistent with experimental observations. (c) Traditional 2-D incommensurate modulation structure with $\mathrm{q} 1=\mathrm{qp}$ and $\mathrm{q} 2=\mathrm{qs}$.

References

[1] T. Ritschel et al, Nat. Phys. 11 (2015) p. 328. 
[2] B. H. Savitzky et al, Nat. Commun. 8 (2017) p. 1883.

[3] L. J. Wu et al, Microsc. Microanal. 25(S2) (2019) p. 1928.

[4] S. Q. Deng et al, Phys. Rev. Lett. 122 (2019) p. 126401.

[5] The authors acknowledge funding from the U.S. DOE-BES, Materials Sciences and Engineering Division, under Contract No. DESC0012704, Chinese National Natural Science Foundation under Project No. 51390471 and the National 973 Project of China (Project No. 2015CB654902). 